\section{Multiples Myelom}

\section{Carfilzomib bereichert die Therapieoptionen im Rezidiv}

Heute stehen für Patienten mit rezidiviertem multiplem Myelom (MM) mehrere Therapieoptionen zur Verfügung. Bei der Therapiewahl sind Faktoren wie Tiefe und Dauer des Ansprechens auf die vorherige Therapie, die Tumorbiologie, patientenbezogene Risikofaktoren sowie die Anzahl der Rezidive ausschlaggebend, wie Andrzej Jakubowiak, Chicago, USA, im Rahmen eines Symposiums bei der Jahrestagung der European Hematology Association (EHA) ausführte.

Eine neue, vielversprechende Therapieoption stellt der Proteasom-Inhibitor Carfilzomib dar. Die ASPIRE-Studie verglich randomisiert bei 792 Patienten mit rezidiviertem und vorbehandeltem MM die Zugabe von Carfilzomib zur Standardtherapie Lenalidomid und Dexamethason [1].

Der primäre Endpunkt der Studie war das progessionsfreie Überleben (PFS), sekundäre Endpunkte waren die Gesamtüberlebenszeit, das Gesamtansprechen, die Dauer des Ansprechens, die gesundheitsbezogene Lebensqualität (EORTC QLQ-C30 Global Health Status) und die Verträglichkeit. Die Studie erreichte den primären Endpunkt für das PFS (Hazard Ratio (HR) 0,69; 95\%-KI 0,57-0,83; $\mathrm{p}<0,0001)$. Die mediane Zeit bis zur Progression war in der Carfilzomib-Gruppe auf 26,3 Monate verlängert; in der Kontrollgruppe betrug sie nur 17,6 Monate. Das ORR im Carfilzomib-Arm betrug 87,4\% (95\%-KI $83,7-90,5)$ und in der Kontrollgruppe 66,9\% (95\%-KI 62,0-71,5). Die häufigsten, behandlungsbedingten schwerwiegenden Nebenwirkungen ( $\geq$ Grad 3) waren Neutropenie $(29,6 \%$ vs. $26,5 \%)$, Anämie ( $17,9 \%$ vs. $17,2 \%)$ und Thrombozytopenie (16,6\% vs. $12,3 \%)$. Nicht- hämatologische Nebenwirkungen aller Schweregrade waren Durchfälle (42,3\% vs. $33,7 \%)$, Erschöpfung (32,9\% vs. $30,6 \%)$ und Husten $(28,8 \%$ vs. $17,2 \%)$. Die häufigsten schwerwiegenden nichthämatologischen $\mathrm{Ne}$ benwirkungen waren Pneumonien (12,5\% vs. $10,5 \%)$, Hypokaliämien ( $9,4 \%$ vs. $4,9 \%)$ und Hypophosphatämien ( $8,4 \%$ vs. $4,6 \%)$. Andere relevante Nebenwirkungen (alle Schweregrade) waren Dyspnoe (22,4\% vs. $18,0 \%$ ), Hypertonie ( $14,3 \%$ vs. $6,9 \%$; $\operatorname{Grad} 3: 4,3 \%$ vs. $1,8 \%)$, akutes Nierenversagen ( $8,4 \%$ vs. $7,2 \%)$, Herzversagen $(6,4 \%$ vs. $4,1 \%)$ und ischämische Herzkrankheit (5,9\% vs. 4,6\%). Periphere Neuropathien (PN) waren mit 17,1\% bzw. 17,0\% vertreten; schwere Verläufe ( $\mathrm{PN} \geq \mathrm{Grad} 3$ ) waren weniger häufig (2,6\% bzw. 3,1\%).

Auch in der Phase-III-Studie ENDEAVOR bestätigte sich der Stellenwert von Carfilzomib beim rezidivierten MM [2]. 929 Patienten erhielten randomisiert entweder Carfilzomib und Dexamethason (Kd) oder Bortezomib und Dexamethason (Vd). Die beim Jahreskongress der amerikanischen Krebsgesellschaft ASCO vorgestellte geplante Interimsanalyse zeigte, dass das mediane PFS im Kd-Arm signifikant länger war als im Vd-Arm (18,7 vs. 9,4 Monate; $\mathrm{HR}=0,53 ; \mathrm{p}<0,0001)$. Zudem war die ORR-Rate im Carfilzomib-Arm signifikant höher (76.9\% vs. $62.6 \%$; p < 0,0001). $54,3 \%$ vs. $28,6 \%$ der Patienten hatten eine «very good partial response or better», $12,5 \%$ vs. $6,2 \%$ hatten unter der Carfilzomib-Kombination eine Komplettremission. Die Nebenwirkungen im Carfilzomib-Arm waren etwas höher als im Bortezomib-Arm, aber insgesamt gering ausgeprägt. Höhergradige Nebenwirkungen unter Carfilzomib ( $\geq$ Grad 3 ) umfassten Bluthoch- druck (8,9\%), Dyspnoe (5,6\%), Herzversagen $(4,8 \%)$ und akutes Nierenversagen (4.1\%). Die Autoren hatten bei der Präsentation beim ASCO den Schluss gezogen, dass die Kombination von Carfilzomib und Dexamethason der Kombination von Bortezomib und Dexamethason im Hinblick auf das PFS signifikant überlegen war und zudem mit einem positiven Nutzen-Risiko-Profil aufwarten konnte. Insgesamt befanden die Autoren, dass Carfilzomib die beste Substanz für diese Gruppe von Patienten sein könnte.

Nach Meinung von Jakubowiak bestätigen diese Studiendaten erneut, dass das MM durch die verbesserten Therapieoptionen mehr und mehr zu einer chronischen Erkrankung wird. In den USA wurde Carfilzomib $\left(\right.$ Kyprolis ${ }^{\circledR}$ ) als zweiter Proteasom-Inhibitor bereits 2012 für die Therapie des rezidivierten MM zugelassen, die Zulassung in Europa ist beantragt.

\section{Dr. Petra Ortner, München}

\section{Quelle}

Amgen Symposium «Charting new depths in the treatment of multiple myeloma», EHA in Wien, 11.6.15

\section{Literatur}

1 Stewart AK et al.: N Engl J Med 2015;372:142-152.

2 Dimopoulos MA et al.: J Clin Oncol 2015;33(suppl): abstr 8509.

Weitere Informationen bei

Amgen $\mathrm{GmbH}$

Hanauer Str. 1, 80992 München

Tel. +49 89 149096-0

www.amgen.com

\title{
PharmaTicker+++ PharmaTicker+++ PharmaTicker+++ PharmaTicker+++
}

Mundipharma. Bendamustin-basierte Behandlungsschemata gelten bereits als Standardtherapie in der First-Line-Behandlung behandlungsbedürftiger Patienten mit indolenten Non-Hodgkin-Lymphomen und chronischer lymphatischer Leukämie. Aktuelle Studiendaten zu vorbehandelten Patienten, vorgestellt auf dem ASCO und dem EHA 2015, bestätigen wiederum, dass sich die Substanz aufgrund ihrer Effektivität und guten Verträglichkeit auch hervorragend als Kombinationspartner für neue Substanzen bei hämatologischen Neoplasien eignet.

Pomme-med GmbH

Carina Jung / jung@pomme-med.de
Janssen. Die EMA hat den Bruton-Tyrosinkinase(BTK)-Inhibitor Ibrutinib (Imbruvica ${ }^{\circledR}$ ) am 8. Juli 2015 zur Behandlung von erwachsenen Patienten mit Morbus Waldenström (MW) zugelassen. Die Zulassung bezieht sich auf die Behandlung von Patienten, die mindestens eine vorangehende Therapie erhalten haben, sowie auf die Erstlinienbehandlung von Patienten mit MW, die für eine Chemoimmuntherapie nicht geeignet sind. Ibrutinib stellt die 1. in Europa zugelassene zielgerichtete Therapie dieser seltenen Krebserkrankung dar.

FleishmanHillard Germany GmbH / Eva Biesenbach eva.biesenbach@fleishmaneurope.com
Abbvie. Im Rahmen des ASCO 2015 wurden neueste Ergebnisse zweier Phase I-Studien für das Prüfpräparat Venetoclax (ABT 199), deren Ansprechraten den möglichen Therapienutzen für die Therapie des multiplen Myeloms aufzeigen, präsentiert. Darüber hinaus wurden Phase-II-Studiendaten für Veliparib (ABT 888) zur Behandlung von Patienten mit nichtkleinzelligem Lungenkarzinom (NSCLC) vorgestellt, auf deren Basis das Unternehmen eine Phase-III-Studie initiierte.

AbbVie Deutschland GmbH \& Co. KG

Maren Nienstedt

maren.nienstedt@abbvie.com

\section{KARGER}




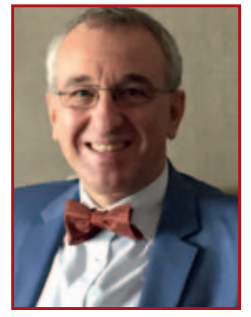

Prof. Dr. med. Wolfgang Schütte, Chefarzt der Klinik für Innere Medizin II, Krankenhaus MarthaMaria Halle-Dölau, Halle (Saale)

Herr Professor Schütte, wie beurteilen Sie die Ergebnisse der zulassungsrelevanten Studie für nab-Paclitaxel (ABRAXANE $\left.{ }^{\circledR}\right)$ in der Indikation des fortgeschrittenen nichtkleinzelligen Lungenkarzinoms (NSCLC)?

In der Studie von Socinski et al. [1] zeigten sich bei den Patienten mit fortgeschrittenem NSCLC eindrucksvolle Ansprechraten im nab-Paclitaxel-plus-Carboplatin-Studienarm. In der Gesamtpopulation lagen diese mit $33 \%$ versus 25\% signifikant höher als im Studienarm mit konventionellem Paclitaxel plus Carboplatin. Interessant war auch die Subgruppenanalyse der Patienten mit Plattenepithelkarzinom. Die Auswertung ergab ein signifikant höheres Therapieansprechen mit nab-Paclitaxel von $41 \%$ versus $24 \%$ [1]. Warum die Remissionsrate beim Plattenepithel so viel besser als beim Adenokarzinom war, muss noch abgeklärt werden. In Bezug auf das Gesamtüberleben fanden sich jedoch keine Unterschiede zwischen Adenound Plattenepithelkarzinom.

Ein besonders hoher Nutzen in puncto Gesamtüberleben zeigte sich bei älteren Patienten, hier speziell in der Gruppe der Patienten ab 70 Jahre. Die Patienten im Studienarm mit nab-Paclitaxel plus Carboplatin profitierten von einem deutlich günstigeren Nebenwirkungsspektrum und einem signifikanten Überlebensvorteil von 19,9 Monaten im Vergleich zu 10,4 Monaten unter konventionellem Paclitaxel plus Carboplatin [1]. Dieser Überlebensvorteil wurde im Studienarm mit nab-Paclitaxel plus Carboplatin auch dann beibehalten, wenn das Alter auf $\geq 60$ Jahre heruntergesetzt wurde. Hier waren es 13,8 Monate mit dem nab-Paclitaxel-Regime versus 11,0 Monate mit konventionellem Paclitaxel plus Carboplatin [2].

Haben Sie bereits Erfahrungen mit nab-Paclitaxel gemacht?

In unserer Klinik haben wir schon einige Patienten mit nab-Paclitaxel behandeln können, nämlich alle diejenigen, für die früher Taxol in der Erstlinientherapie infrage gekommen wäre. Hier haben wir sozusagen einfach einen Austausch gemacht. Das Therapieregime mit nab-Paclitaxel wurde von den Patienten außerordentlich gut vertragen - so wie wir dies nach den Ergebnissen der Zulassungsstudie auch erwartet hatten.

Was zeichnet eine Therapie mit nab-Paclitaxel im klinischen Alltag aus?

Für den klinischen Alltag von besonderer Bedeutung ist das deutlich bessere Nebenwirkungsspektrum, das sich im nab-Paclitaxel-Studienarm gezeigt hat, vor allem die deutlich reduzierte Neurotoxizität. Dies ist aus zwei Gründen von besonderer Bedeutung: Zum einen beeinträchtigen die Folgen der Neurotoxizität wie Schmerzen sowie Störungen von Sensibilität und des Tastempfindens sehr stark die Lebensqualität. Zum anderen sind die Nervenschädigungen im Gegensatz zu den hämatologischen Veränderungen meist nicht reversibel. Deswegen ist die Neurotoxizität sehr gefürchtet. Der eindeutige Vorteil von nab-Paclitaxel gegenüber den herkömmlichen Taxanen ist ganz klar die signifikante Reduktion der Toxizität insgesamt und der Neurotoxizität im Besonderen. Dies war in der Studie von Socinski über alle Therapiezyklen hinweg eindeutig festzustellen [1].

Wie erklärt man sich die bessere Verträglichkeit von nab-Paclitaxel?

Nab-Paclitaxel ist die bisher erste und einzige albuminbasierte Nanotechnologieformulierung von Paclitaxel. Das «nab» in nab-Paclitaxel steht für «nanoparticle albumin-bound». Die innovative nab-Technologie macht sich die natürlichen Eigenschaften von Albumin zunutze, wodurch es zu einer besseren und schnelleren Verteilung der Substanz im Organismus kommt. Es resultiert eine gezielte Aufnahme und Anreicherung der Wirksubstanz in den Tumor. Ein weiterer Vorteil der nab-Technologie: Auf die bei konventionellem Paclitaxel notwendigen Lösungsmittel kann bei nab-Paclitaxel verzichtet werden. Dies ist ein großer Vorteil, denn der Lösungsvermittler Cremophor kann schwere allergische Reaktionen auslösen und hat $\mathrm{zu}-$ sätzlich eine neurotoxische Wirkung. Dadurch, dass Cremophor nicht mehr notwendig ist, ist vermutlich auch die Neurotoxizität geringer.
Hat der Wegfall von Lösungsvermittlern auch Auswirkungen auf das klinische Handling?

Der wichtigste Punkt ist, dass die Prämedikation mit Steroiden und Antihistaminika entfällt, was die Verabreichung von nab-Paclitaxel unproblematischer macht. Zum einen ist das Risiko allergischer Reaktionen deutlich reduziert und zum anderen entfallen die Nebenwirkungen der Prämedikation. Insbesondere die Steroide können schwere Nebenwirkungen verursachen. Die in der Prämedikation eingesetzten Antihistaminika sind ebenfalls nicht unproblematisch, denn sie machen müde und die Patienten können im Anschluss an die Behandlung nicht Auto fahren. Der Wegfall der Prämedikation macht die Gabe von nab-Paclitaxel einfacher und ist auch für den Patienten sicherer.

Haben Sie ein konkretes Patientenbild vor Augen, bei dem Sie nab-Paclitaxel vorzugsweise einsetzen werden?

Platinhaltige Verbindungen in Kombination mit einem Taxan sind Standard in der Chemotherapie des fortgeschrittenen NSCLC. Mit nab-Paclitaxel plus Carboplatin steht uns jetzt eine effektive und neue First-Line-Therapie für Patienten mit fortgeschrittenem NSCLC zur Verfügung. Wann immer ein Regime mit platinhaltigen Verbindungen in Kombination mit einem Taxan indiziert ist, dann sollten wir jetzt auf nab-Paclitaxel setzen.

Vielen Dank für das Gespräch.

Dr. med. Kirsten Westphal, München

\section{Literatur}

Socinski MA et al.: J Clin Oncol 2012;30:2055-2062 Langer CJ et al.: Br J Cancer 2015;113:20-29.

Weitere Informationen bei Prof. Dr. med. Wolfgang Schütte Chefarzt der Klinik für Innere Medizin II Krankenhaus Martha-Maria Halle-Dölau Röntgenstraße 1

06120 Halle (Saale)

Innere-Medizin2.Halle@Martha-Maria.de 


\section{Fortgeschrittenes malignes Melanom}

\section{Checkpoint-Blockade 2.0}

Nachdem die Checkpoint-Blockade mit dem CTLA-4-Inhibitor Ipilimumab (Yervoy ${ }^{\circledR}$ ) erstmals einen deutlichen Überlebenszeit-Gewinn für Patienten mit fortgeschrittenem malignem Melanom (MM) möglich gemacht hat, wurde jetzt eine weitere Immuntherapie mit neuem Wirkansatz in der Checkpoint-Blockade zugelassen: Nivolumab (Opdivo $\left.{ }^{\circledR}\right)$ hemmt die Interaktion des Rezeptors "Programmed Death 1" (PD-1) mit seinen Liganden PD-L1 und PD-L2. Prof. Dr. Dirk Schadendorf, Essen, bezeichnete die beschleunigte Zulassung in Europa als erneuten Meilenstein in der Therapie des MM.

\section{Dramatisch verlängertes Überleben}

Die Zulassung von Nivolumab durch die Europäische Kommission beruht auf den Ergebnissen der Phase-III-Studien CheckMate -066 mit therapienaiven und CheckMate-037 mit vorbehandelten Patienten mit fortgeschrittenem MM [1, 2]. In CheckMate -066 wurden die Wirksamkeit und Sicherheit der Therapie mit dem PD-1-Inhibitor Nivolumab (3 mg/kg Körpergewicht alle 2 Wochen; $\mathrm{n}=210$ ) mit einer Chemotherapie mit Dacarbazin $\left(1000 \mathrm{mg} / \mathrm{m}^{2}\right.$ alle 3 Wochen; $\mathrm{n}$ $=208$ ) bei Patienten mit therapienaivem fortgeschrittenem MM verglichen [1]. Es zeigte sich ein klarer Überlebensvorteil für die PD-1Blockade: Die 1-Jahres-Überlebensrate betrug unter Nivolumab 73\% im Vergleich zu 42\% unter Dacarbazin (Hazard Ratio (HR) 0,42; 95\%-Konfidenzintervall (KI) $0,25-0,73$; p < 0,0001). Auch die objektive Ansprechrate (ORR) war bei der Therapie mit Nivolumab signifikant höher als mit Dacarbazin (40 vs. 14\%; p < 0,0001). Zudem wurde die Behandlung unter Nivolumab seltener abgebrochen als unter $\mathrm{Da}$ carbazin ( 6,8 vs. $11,7 \%)$, und es traten weniger therapiebedingte unerwünschte Ereignisse (UE)
Oncol Res Treat 38 | 9 | 15

vom Grad 3/4 auf (11,7 vs. 17,6\%). Die häufigsten mit Nivolumab assoziierten UE waren Fatigue (20\%), Juckreiz (17\%) und Übelkeit (16,5\%). Aufgrund der laut Schadendorf «dramatischen» Wirkung wurde die Studie vorzeitig beendet, damit auch die Patenten der Kontrollgruppe den PD1-Hemmer erhalten konnten.

\section{Vorteil auch bei vorbehandelten Patienten}

Die offene Phase-III-Studie CheckMate -037 verglich randomisiert und kontrolliert Nivolumab ( $n=272)$ mit einer vom Prüfarzt gewählten Chemotherapie (entweder Dacarbazin alleine oder Carboplatin plus Paclitaxel; $\mathrm{n}=133$ ) bei Patienten mit fortgeschrittenem MM, die mit Ipilimumab und - falls BRAF-positiv - mit einem BRAF-Inhibitor vorbehandelt waren [2]. In einer geplanten Interimsanalyse lag die ORR bei den mit Nivolumab behandelten Patienten bei 32\% (95\%-KI 23,5-40,8), in der Chemotherapiegruppe bei $11 \%(95 \%-K I ~ 3,5-23,1)$. Die Toxizitätsdaten von Nivolumab waren konsistent mit denen der CheckMate -066-Studie. Zudem war das Ansprechen unabhängig vom BRAF-Mutationsstatus und von der PD-L1-Expression. Schadendorf betonte, dass wenn Patienten ein Ansprechen auf die Therapie zeigen, dies oft über Monate besteht. Er sagte: «Jetzt ist die Vision Langzeitüberleben oder gar klinische Heilung.» Und Prof. Dr. Axel Hauschild, Kiel, begrüßte, dass die Zulassung von Nivolumab nicht auf die Zweitlinie beschränkt ist, sondern gemäß der Studiendaten in allen Linien eingesetzt werden kann. Die deutschen Leitlinien werden in der Aktualisierung Ende des Jahres die neue Option der PD-1-Blockade berücksichtigen.

\section{Chance auch für andere solide Tumoren}

Ein großes Potenzial hat die PD-1-Blockade auch beim Lungenkarzinom, berichtete Prof.
Dr. Wolfgang Herr aus Regensburg. Eine Phase-III-Studie verglich die Wirksamkeit und Sicherheit von Nivolumab mit Docetaxel beim vorbehandelten fortgeschrittenen refraktären nicht-kleinzelligen Lungenkarzinom [3]. Dabei verlängerte die Therapie mit Nivolumab das Gesamtüberleben bei diesen Patienten mit schlechter Prognose um im Median 3,2 Monate (9,2 vs. 6,0 Monate), das 1-Jahres-Überleben betrug im Nivolumab-Arm 42\%, im Docetaxel-Arm 24\% (HR für Tod 0,59; 95\%-KI 0,44$0,79 ; \mathrm{p}<0,001)$. «Das ist beeindruckend», meinte Herr, «aber noch viel wichtiger ist, dass wir unseren Patienten nicht zu viel zumuten.» Im Nivolumab-Arm traten sehr viel seltener schwere UE auf als im Chemotherapie-Arm (7 vs. 55\%). Zwischenzeitlich wurde Nivolumab auch beim fortgeschrittenen Lungenkarzinom zugelassen: Seit dem 20. Juli 2015 steht der PD-1-Inhibitor Patienten mit lokal fortgeschrittenem oder metastasiertem nichtkleinzelligen Lungenkarzinom mit plattenepithelialer Histologie nach Chemotherapie zur Verfügung.

Friederike Klein, München

\section{Quelle}

Pressekonferenz von Bristol-Myers Squibb «Mit OPDIVO ${ }^{\circledR}$ von der Vision zur Wirklichkeit. Eine neue Zeit für Patienten mit metastasiertem Melanom» am 26. Juni 2015 in München

\section{Literatur}

1 Robert C et al.: N Engl J Med 2015;372:320-330.

2 Weber J et al.: Lancet Oncology 2015;16:375-384.

3 Brahmer J et al.: N Engl J Med 2015;373:123-135.

Weitere Informationen bei Bristol-Myers Squibb GmbH \& Co. KGaA Ina Fürholzer ina.fuerholzer@bms.com www.bms-onkologie.de

\section{Nivolumab BMS ${ }^{\circledR}$ (Nivolumab) erhält EU-Zulassung beim lokal fortgeschrittenen oder metastasierten nichtkleinzelligen Lungenkarzinom mit plattenepithelialer Histologie nach Chemotherapie}

Die Europäische Kommission hat am 20. Juli 2015 Nivolumab BMS ${ }^{\circledR}$ (Nivolu$\mathrm{mab})$ zur Behandlung von erwachsenen Patienten mit lokal fortgeschrittenem oder metastasiertem nichtkleinzelligem Lungenkarzinom mit plattenepithelialer Histologie nach vorheriger Chemotherapie zugelassen [1]. Diese Zulassung markiert den ersten großen Therapiefortschritt in der Behandlung des nichtkleinzelligen Plattenepithelkarzinoms der Lunge in der EU seit über einem Jahrzehnt [2]. Nivolumab ist zudem der erste PD-1-Inhibitor, der einen Gesamtüberlebensvorteil beim vorbehandelten metastasierten nichtkleinzelligen Plattenepithelkarzinom der Lunge gezeigt hat [2]. Die erteilte Zulassung gilt für alle 28 EU-Mitgliedsländer. «Durch die EU-Zulassung von Nivolumab haben Patienten in Deutschland und Europa zum ersten Mal seit 10 Jahren Zugang zu einer völlig neuen Behandlungsoption beim fortgeschrittenen nichtkleinzelligen Plattenepithelkarzinom der Lunge», sagte Han Steutel, Ge- schäftsführer von Bristol-Myers Squibb in Deutschland. «Diese Zulassung ist die 2., die Bristol-Myers Squibb für Nivolumab in Europa innerhalb von weniger als 2 Monaten erhält. Das macht uns sehr stolz, denn diese Zulassung steht auch exemplarisch für unser unablässiges Engagement und unser Bestreben, die Lebenserwartung und Lebensqualität von Patienten mit fortgeschrittenen Krebserkrankungen nachhaltig zu verändern.»

\section{Literatur}

1 Nivolumab BMS SMPC; 14. Mai 2015.

2 Datamonitor Healthcare. Non-Small Cell Lung Cancer Marketed Drugs (letzter Zugriff: 8. Juli 2015).

Bristol-Myers Squibb GmbH \& Co. KGaA Ina Fürholzer / ina.fuerholzer@bms.com 


\section{PharmaNews}

Oncol Res Treat 38 | 9 | 15

20. Kongress der European Hematology Association (EHA)

\section{Vielversprechende Zukunft der CLL- und FL-Therapie}

Ein Thema, das auch im Rahmen des 20. Kongresses der European Hematology Association (EHA) in Wien große Aufmerksamkeit erhielt, war die stetige Weiterentwicklung neuer zielgerichteter Therapieoptionen zur Behandlung der chronischen lymphatischen Leukämie (CLL) und des follikulären Lymphoms (FL). So wurden auch auf dem Satellitensymposium der Firma Gilead Sciences aktuelle Daten aus klinischen Studien präsentiert. Die Experten zeigten auf, wie diese in die klinische Praxis übertragen werden können und diskutierten die künftige Veränderung bestehender Therapieschemata.

Die kürzliche Einführung von gezielten BCR-Signalweg-Inhibitoren wie z.B. Idelalisib könnte eine neue Ära in der Behandlung von FL-Patienten einläuten, erklärte Professor Gilles Salles, Hospital Centre Lyon-Sud, Frankreich. In der Studie 101-09 wurde Idelalisib als Monotherapie beim doppelt refraktären FL untersucht. Mehr als 55\% der Patienten zeigten unter der Behandlung mit Idelalisib ein Therapieansprechen, obwohl zuvor unter Rituximab und unter einem Alkylanz kein Ansprechen vorlag [1-3]. Derzeit werden verschiedene Kombinationsmöglichkeiten von BCR-Signalweg-Inhibitoren mit weiteren Substanzen zur Therapie des FL getestet. Bei der CLL konnte durch die zielgerichtete Inhibition die behandlungsbezogene Toxizität verringert und somit die Therapiemöglichkeiten gerade bei unfitteren Patienten erweitert werden. Auch Hochrisikopatienten profitieren stark von den neuen Substanzen.

So konnten vergleichbare Therapieergebnisse auch bei Patienten mit verschiedenen genetischen Risikofaktoren beobachtet werden. Beispielsweise lag das progressionsfreie Überleben bei Patienten mit bzw. ohne del(17p)/TP53-
Mutation bei 16,6 bzw. 20,3 Monaten unter Idelalisib plus Rituximab $(\mathrm{p}=0,94)$ [4]. Bei diesen Hochrisikopatienten ist Idelalisib in Kombination mit Rituximab sogar in der Erstlinie zugelassen, wenn die Patienten für eine Chemoimmuntherapie ungeeignet sind. Zudem waren sich die Experten einig: Der Einsatz stark wirksamer Antikörper und Kinaseinhibitoren könnte künftig die Planung einer langfristigen Kontrolle der CLL erlauben.

\section{Rituximab als Wegbereiter}

Rituximab erzielt in Kombination mit einer Chemotherapie sowohl in der Erstbehandlung als auch im Rezidiv bereits starke Ergebnisse bei CLL-Patienten. Für FL-Patienten mit hoher Tumorlast, die nicht oder nicht mehr auf eine vorherige Chemotherapie ansprechen, ist die alleinige Gabe von Rituximab schon gängig. Weitere chemotherapiefreie Ansätze, wie die Kombination von Rituximab mit bereits bekannten oder neuen Wirkstoffen, wurden in verschiedenen Studien untersucht. Dazu zählt etwa die Kombination von Rituximab und Idelalisib bei CLL-Patienten. In der doppelblinden Phase-III-Studie 312-0116 wurde die Behandlung bei stark komorbiden Patienten mit einem Rezidiv im Vergleich zu Rituximab mit Placebo untersucht. Die Kombination mit Idelalisib zeigte ein signifikant längeres Gesamtüberleben (medianes Gesamtüberleben, noch nicht erreicht vs. 20,8 Monate; $\mathrm{p}<0,0001$ ) [4].

Neue Kombinationstherapien in der klinischen Prüfung

Nach dem Beispiel der Kombination von Rituximab und BCR-Signalweg-Inhibitoren wird nun auch die Kombination von BCR-Signalweg-Inhibitoren mit weiteren monoklonalen CD20-Antikörpern überprüft. Vielversprechende Ergebnisse zeigt beispielsweise die klinische Phase-III-Studie 119, die die Kombination aus Ofatumumab mit Idelalisib bei vorbehandelten CLL-Patienten untersucht. Im Vergleich zur Ofatumumab-Monotherapie zeigt die Kombination mit Idelalisib eine Reduktion des Risikos für eine Erkrankungsprogression oder Tod um 73\% (Hazard Ratio 0,27; $\mathrm{p}<0,0001)[5]$.

\section{Quellen}

Satellitensymposium «The changing landscape in CLL and follicular lymphoma: How do new targeted therapies impact patients' lives?», Gilead Sciences, 11.6.2015, Wien

Satellitensymposium «Updates in Hematology: Can we further improve CLL and FL patients' lives? Recent advances and case studies», Gilead Sciences, 11.6.2015, Wien

\section{Literatur}

1 Gopal AK et al.: ASH 2014; abstr 1708: Posterpräsentation.

2 Salles G et al.: ASCO 2015; abstr \#346.

3 Zinzani P et al.: EHA 2015, abstr \#689.

4 Sharman JP et al. Blood 2014:124:33

5 Jones JA et al.: J Clin Oncol 2015;33(suppl): abstr \#7023.

Weitere Informationen bei

Dr. Diane Langenbacher-Nießing

Gilead Sciences GmbH

Fraunhoferstr. 17

82152 Martinsried

Diane.Langenbacher@gilead.com

\section{CHMP empfiehlt EU-Zulassung der Kombinationstherapie mit Panobinostat für} vorbehandelte Patienten mit Multiplem Myelom

Der Ausschuss für Humanarzneimittel (CHMP) der Europäischen Arzneimittelagentur empfiehlt die EU-Zulassung von Panobinostat in Kombination mit Bortezomib und Dexamethason zur Behandlung von erwachsenen Patienten mit Multiplem Myelom, die mindestens 2 vorausgegangene Therapien, darunter Bortezomib und eine immunmodulatorische Substanz, erhalten haben [1]. Panobinostat ist in den USA in dieser Indikation bereits seit Februar 2015 unter dem Handelsnamen Farydak ${ }^{\circledR}$ zugelas- $^{-}$ sen [2]. Sobald das CHMP-Votum von der EU-Kommission bestätigt wird, steht dieser Patientengruppe auch in Europa mit Panobinostat der 1. Vertreter der neuen Substanzklasse der pan-HDAC(Histon-Deacetylasen)-Inhibitoren zur Verfügung. Dies bedeutet einen wichtigen Fortschritt in der Behandlung von Patienten mit rezidiviertem oder rezidiviertem und refraktärem Multiplem Myelom, die eine besonders ungünstige Prognose aufweisen. Die Wirkung von Panobinostat beruht auf einer breiten Hemmung aller Klassen von HDAC, wodurch es an den Zielmolekülen zu einer verstärkten Acetylierung kommt. Dies kann zur Proliferationshemmung und Apoptose von Myelomzellen führen.

\section{Literatur}

1 CHMP LBH589 Summary of Opinion.

2 FDA News Release February 2015. www.fda.gov/NewsEvents/Newsroom/ PressAnnouncements/ucm435296.htm/ (letzter Zugriff am 20. Juni 2015).

\section{Quelle}

Novartis Onocology Pressemitteilung «Farydak ${ }^{\circledR}$ von Novartis erhält Positive Opinion: Neuer Wirkmechanismus in der Kombinationstherapie vorbehandelter Patienten mit Multiplem Myelom in Aussicht», 10. Juli 2015, Autor: Novartis Pharma GmbH 


\section{Cobimetinib und Vemurafenib}

\section{Prüfkombination überzeugt bei Patienten mit fortgeschrittenem Melanom}

Kombinationstherapien zählen zu den wirksamsten Strategien, um die Prognose von Krebspatienten weiter zu verbessern. Beim fortgeschrittenen $\mathrm{BRAF}^{\mathrm{V} 600}$-Melanom bestätigen dies erneut aktuelle Daten zur Kombination von Cobimetinib plus Vemurafenib (Zelboraf ${ }^{\circledR}$ ), die erstmals auf der 51. Jahrestagung der American Society of Clinical Oncology (ASCO) in Chicago, IL, USA, präsentiert wurden: So verlängerte die zusätzliche Behandlung mit Cobimetinib in der Phase-III-Studie coBRIM das mediane progressionsfreie Überleben gegenüber der Vemurafenib-Monotherapie signifikant um mehr als 5 Monate [1].

Die vorgestellten Daten der coBRIM-Studie
Oncol Res Treat 38 | 9 | 15

untermauern das große Potenzial der kombinierten Behandlung mit Cobimetinib und Vemurafenib bei Patienten mit fortgeschrittenem BRAF ${ }^{\mathrm{V} 600}$-Mutation-positivem Melanom. In die internationale, randomisierte, doppelblinde und placebokontrollierte Phase-III-Studie wurden 495 therapienaive Patienten mit $\mathrm{BRAF}^{\mathrm{V} 600}$-Mutation-positivem inoperablem, lokal fortgeschrittenem oder metastasiertem Melanom eingeschlossen, die entweder mit Cobimetinib plus Vemurafenib oder mit Vemurafenib alleine behandelt wurden. Das zentrale Ergebnis der aktuellen Analyse vom ASCO: Patienten, die in der Studie die Kombinationstherapie erhielten, erreichten im Median ein progressionsfreies Überleben von über einem Jahr (12,3 Monate) - im Vergleich dazu blieben die Patienten, die Vemurafenib als Monotherapie erhielten, nur 7,2 Monate ohne ein Fortschreiten der Erkrankung (Hazard Ratio 0,58;
95\%-Konfidenzintervall 0,46-0,72). Auch die objektive Ansprechrate lag in der Kombinationsgruppe höher (70 vs. 50\%). Zudem erreichten mehr Patienten unter Cobimetinib/Vemurafenib eine komplette bzw. partielle Remission als unter der Monotherapie (16 vs. 11\% bzw. 54 vs. 40\%) [1]. Auf der Basis der positiven Analyse stellte die Roche Pharma AG den Zulassungsantrag für die Kombinationstherapie Cobimetinib/Vemurafenib für diese Patientengruppe bei der Europäischen Zulassungsbehörde.

\section{Literatur}

1 Larkin J et al.: J Clin Oncol 2015;33(suppl): abstr \#9006.

Weitere Informationen bei

Roche Pharma AG

Ulla Satzger

ulla.satzger@roche.com

\section{Therapie älterer AML-Patienten ab 65 Jahren}

\section{Praktische Erfahrungen mit Dacogen ${ }^{\circledR}$}

die AML in eine proliferative Erkrankung umgeschlagen ist, hat man eine relativ grobe zytostatische Behand-

Seit rund 2,5 Jahren steht erwachsenen Patienten ab 65 Jahren mit neudiagnostizierter de novo oder sekundärer akuter myeloischer Leukämie (AML) gemäß WHO-Klassifikation, für die eine Standardinduktionstherapie nicht infrage kommt, mit Decitabin $\left(\right.$ Dacogen $\left.{ }^{\circledR}\right)$ eine epigenetische Therapieoption zur Verfügung [1]. Mittlerweile ist es eine etablierte Substanz bei der Behandlung dieser Patientengruppe. Professor Dr. Karl-Anton Kreuzer, Köln, berichtet über seine praktischen Erfahrungen mit Decitabin.

Welche Therapieoptionen älteren Patienten vor der Zulassung von epigenetischen Therapien zur Verfügung standen, erläutert Kreuzer: «Man hat in vielen Fällen nicht kausal behandelt, sondern eine rein supportive Therapie angewandt. Wenn lung durchgeführt, die weniger zum Ziel hatte, unbedingt eine Remission zu erreichen, sondern eher dazu diente, erkrankungsbedingte Komplikationen zu verhindern.» Die AML ist eine hämatologische Neoplasie mit einer schlechten Prognose. Dies gilt in besonderem Maße für Patienten, die ein höheres Alter ( $\geq 65$ Jahre), einen schlechten Allgemeinzustand und/oder 3 oder mehr Chromosomenaberrationen aufweisen [2]. Decitabin wird in die hypermethylierte DNA eingebaut und verhindert dort eine weitere Methylierung der Erbsubstanz. So unterstützt es die Reaktivierung von wichtigen Tumorsuppressor-Genen und kann das Verhältnis zwischen Zellproliferation und natürlichem Zelltod verbessern und damit das Tumorwachstum verlangsamen [3]. «Epigenetische Therapien haben das
Ziel, die Zellen genetisch so zu modifizieren, dass beispielsweise ein Tumorsuppressor-Gen wieder aktiviert wird,» beschreibt Kreuzer den epigenetischen Wirkansatz. In den vergangenen 2 Jahren habe sich Decitabin als gut verträglich bewährt, erklärte Kreuzer. Seine Erfahrung ist, dass die intravenöse Applikationsform als wesentlich angenehmer empfunden wird als zum Beispiel eine subkutane Therapie, die zu unangenehmen lokalen Reaktionen führen könne.

\section{Literatur \\ 1 Aktuelle Dacogen ${ }^{\circledR}$-Fachinformation. \\ 2 Serve $\mathrm{H}$ et al.: Prognose; www.onkodin.de/e2/e51675/ e54836 (letzter Zugriff 27.04.2015). \\ 3 Oki Y et al.: Crit Rev Oncol Hematol 2007;61:140-152.}

Weitere Informationen bei

FleishmanHillard Germany GmbH

Teresa Hirth

teresa.hirth@fleishmaneurope.com

\section{Übelkeit und Erbrechen bei Chemotherapie (CINV) - Palonosetron-basierte Antiemese auch bei Carboplatin-basierter Chemotherapie und bei pädiatrischen Patienten im Säuglingsalter hochwirksam}

Bei dem diesjährigen ASCO stand in den Sessions zur Supportivtherapie die Prävention von chemotherapieinduzierter Übelkeit und Erbrechen (CINV) im Fokus. Es wurden unter anderem neue Studiendaten zum Einsatz der oralen Fixkombination Netupitant/Palonosetron (NEPA; Akynzeo ${ }^{\circledR}$ ) bei erwachsenen Patienten unter Carboplatin-basierter Chemotherapie vorgestellt. Eine weitere Untersuchung befasste sich mit dem Einsatz von Palonosetron (PALO; Aloxi $^{\circledR}$ ) bei Kindern ab einem Alter von 64 Tagen, die eine Chemotherapie erhalten müssen. Beide Untersuchungen belegen die hohe antiemetische Wirksamkeit und Sicherheit des derzeit effektivsten $5-\mathrm{HT}_{3}$-Rezeptorantagonisten (5- $\left.\mathrm{HT}_{3}-\mathrm{RA}\right)$ Palonosetron in verschiedenen Settings. Mit der oralen Fixkombination aus dem $\mathrm{NK}_{1}$-Rezeptorantagonisten ( $\left.\mathrm{NK}_{1}-\mathrm{RA}\right)$ Netupitant und dem $5-\mathrm{HT}_{3}$-RA Palonosetron ist aktuellen Studiendaten zufolge eine hocheffektive Prävention der CINV über den ge- samten Chemotherapiezyklus und über multiple Chemotherapiezyklen möglich [1-4]. Die Kombination wurde Ende Mai 2015 in der EU zur antiemetischen Prävention von Übelkeit und Erbrechen bei hochemetogener Cisplatin-basierter Chemotherapie und moderat emetogener Chemotherapie zugelassen [5].

\section{Literatur}

1 Hesketh PJ et al.: Ann Oncol 2014;25:1340-1346.

2 Aapro M et al.: Ann Oncol 2014;25:1328-1333.

3 Karthaus M et al.: ASH Annual Meeting Abstracts; Blood 2014;124:4821.

4 Gralla R et al.: Ann Oncol 2014;25:1333-1339.

5 www.ema.europa.eu/docs/en_GB/document_library/Summary_of_opinion/ human/003728/WC500184907.pdf.

pomme-med GmbH / Carina Jung / jung@ pomme-med.de 